\title{
Die Beprobung von Jahresschmutzfrachten in einem Mischwasser- und einem Niederschlagswasserkanal bei Regenwetter
}

\author{
Günter Gruber · Markus Pichler · Thomas Hofer · Roman Maier · Manfred Clara
}

Online publiziert: 13. Februar 2020

(c) Der/die Autor(en) 2020

Zusammenfassung Niederschlagswasser- und Mischwassereinleitungen können für einige Spurenstoffe aus dem urbanen Bereich der dominierende Eintragspfad in die Gewässer sein. Im Rahmen eines österreichischen Forschungsprojekts wurden an einer Niederschlagswasserkanaleinleitungsstelle in ein Gewässer sowie an einem Mischwasserüberlauf zwei mengenproportionale Jahresmischproben generiert, um die daraus resultierenden ein- bzw. abgeleiteten Jahresschmutzfrachten an Spurenstoffen zum einen messtechnisch zu bestimmen und zum anderen nach einer detaillierten Analyse der Oberflächenbeschaffenheiten in den Einzugsgebieten und entsprechenden Stoffflussanalysen theoretisch ermittelbaren Stofffrachtpotenzialen aus der Literatur gegenüberzustellen. Die dafür gewählte Probenahmestrategie, die dabei gesammelten Erfahrungen bei der Probenahme und die erzielten Ergebnisse aus den Beprobungen werden im Beitrag beschrieben und diskutiert.

Schlüsselwörter Probenahme . Automatische Probenahme Mengenproportionale Probenahme . Jahresschmutzfrachten · Spurenstoffe . Mischwasser $\cdot$ Niederschlagswasser

Ass.-Prof. DI Dr. G. Gruber $(\bowtie)$. DI M. Pichler, BSc · DI R. Maier, BSc Institut für Siedlungswasserwirtschaft und Landschaftswasserbau,

Technische Universität Graz, Stremayrgasse 10/I, $8010 \mathrm{Graz}$, Österreich

guenter.gruber@tugraz.at

DI T. Hofer, BSc

Ingenieurgemeinschaft DI Bilek \&

DI Krischner GmbH, Krenngasse

9, 8010 Graz, Österreich

DI Dr. M. Clara

Umweltbundesamt, Spittelauer Lände 5, 1090 Wien, Österreich

\section{Sampling of annual pollution} loads in a combined sewer and a stormwater sewer during rainy weather

Abstract Stormwater runoff at sewer overflows (combined sewer overflows and stormwater outlets) can be the dominant path of entry into natural water bodies for some micropollutants from urban areas. Within the scope of an Austrian research project, two annual mixed samples proportional to the annual runoff of an entire sampled year were generated at a stormwater outlet and a combined sewer overflow in order to determine the resulting annual micropollutant loads by measurements and to compare them with theoretically determined potentials for the micropollutant loads from the literature after a detailed analysis of the surface conditions in the catchment areas and corresponding material flow analysis. The chosen sampling strategy, the experience gained during sampling and the results obtained from the sampling are described and discussed in the article.

Keywords Sampling · Automatic sampling · Flow proportional sampling - Yearly pollutant loads . Micropollutants · Combined wastewater $\cdot$ Stormwater

\section{Einleitung}

Niederschlagswasser- und Mischwassereinleitungen können für Spurenstoffe aus dem urbanen Bereich der dominierende Eintragspfad in die Gewässer sein (Clara et al. 2014; Huber et al. 2015; MKULNV 2016; Wicke et al. 2016; Launay 2017). Effektive Niederschlagswasser- und Mischwasserbehandlung sind somit in vielen Fällen entscheidend für die Gewässergüte und damit auch für die Erreichung der Zielsetzungen der EU-Wasserrahmenrichtlinie.
Im Rahmen des Projektes SCHTURM (Spurenstoffemissionen aus Siedlungsgebieten und von Verkehrsflächen, Clara et al. 2014) wurden in Österreich in den Jahren 2012 bis 2014 unterschiedliche Eintragspfade in die Gewässer auf organische und anorganische Spurenstoffe untersucht. Das Ziel dieses Projekts war eine frachtbasierte Bewertung der Emissionen aus Siedlungsgebieten und die Identifikation von Haupteintragspfaden. Die dafür durchgeführten Beprobungen, Spurenstoffuntersuchungen und Frachtabschätzungen basierten auf einigen wenigen (3 bis 5) mengenproportionalen Ereignismischproben in 4 verschiedenen Untersuchungsgebieten. Dabei zeigten die Ergebnisse weder ein eindeutiges Bild, noch war ein Eintragspfad bei allen untersuchten Stoffen dominant.

Das Projekt (T)EMPEST („Erfassung von Emissionen ausgewählter Spurenstoffe aus Kanalsystemen und Handlungsoptionen $\mathrm{zu}$ deren Minderung und Optimierung einer alternativen Nachweismethode für Kunststoffpartikel in Wasserproben") zielt unter anderem auf eine Erweiterung der bestehenden Datenbasis zum Vorkommen ausgewählter Spurenstoffe im Abwasser mit dem Fokus auf Niederschlagswassereinleitungen aus Trennkanalisationen $\mathrm{ab}$, wofür abermals in 3 der 4 SCHTURM-Untersuchungsgebieten Beprobungen in insgesamt 4 Niederschlagswasserkanälen (NWK) und einem Mischwasserkanal (MWK) über ein Jahr lang durchgeführt wurden. Im Unterschied zum SCHTURM-Projekt, wo nur einige wenige Niederschlagsereignisse beprobt wurden, sollten jetzt im (T)EMPEST-Projekt insgesamt 5 mengenproportionale Jahresmischproben generiert werden, um daraus dann die abgeleiteten bzw. in die Gewässer eingeleiteten Jahresstofffrachten messtechnisch abschätzen zu können. Diese messtechnisch ermittelten Jahresfrachten sollen in weiterer Folge 


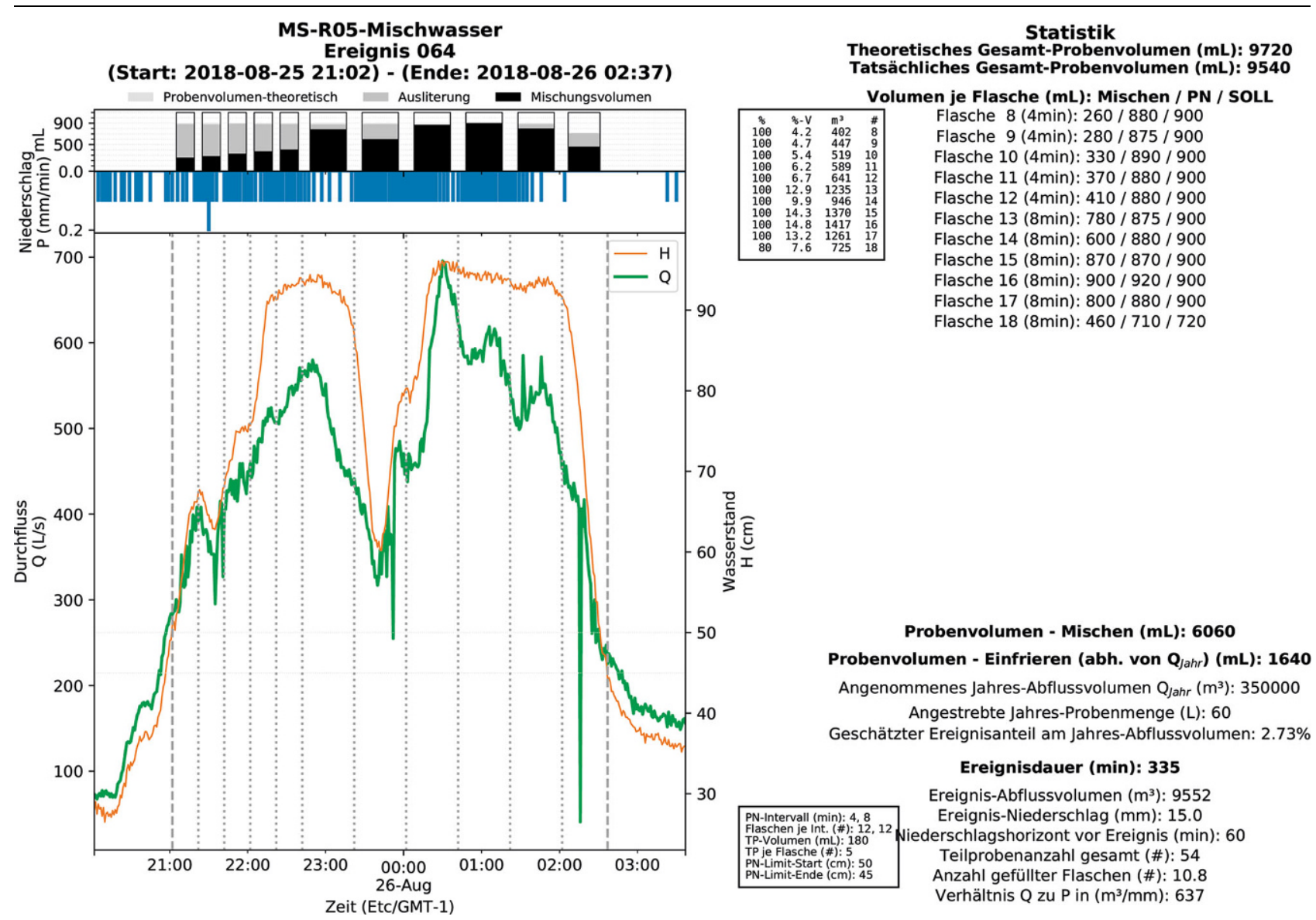

Abb. 1 Beispielhafte Auswertung des MW-Ereignisses 064 an der Probenahmestelle Graz-MÜ-R05 inkl. automatisch generierter Mischungstabelle für das Ereignis und dem einzufrierenden Ereignis-Aliquot für die Jahresmischprobe

nach einer detaillierten Analyse der Oberflächenbeschaffenheiten in den Einzugsgebieten und entsprechenden Stoffflussanalysen theoretisch ermittelbaren Stofffrachtpotenzialen aus der Literatur gegenübergestellt werden.

Für eine messtechnische Abschätzung der Jahresstofffrachten war es dafür an den 5 Kanal-Probenahmestellen erforderlich, möglichst alle abflusswirksamen Niederschlagsereignisse eines Jahres möglichst vollständig mengenproportional zu beproben und entsprechende Aliquote eines jeden beprobten Ereignisses ebenfalls mengenproportional $\mathrm{zu}$ einer Jahresmischprobe $\mathrm{zu}$ vereinigen, die durch kumulatives Einfrieren der Ereignis-Aliquote erzeugt wurde. Aus diesen Jahresmischproben werden schließlich wieder ausgewählte Spurenstoffkonzentrationen bestimmt, die - wie schon im SCHTURM-Projekt - ein ubiquitär persistentes, bioakkumulierendes und toxisches Verhalten aufweisen (sog. uPBTs).

Die dafür gewählte Probenahmestrategie, die dabei gesammelten Erfahrun- gen bei der Probenahme und die erzielten Ergebnisse aus den Beprobungen in einem Niederschlagswasser- und in einem Mischwasserkanal im (T)EMPESTUntersuchungsgebiet Süd in Graz werden in diesem Beitrag beschrieben und diskutiert.

\section{Beschreibung der beiden Probenahmestellen in Graz}

\subsection{Niederschlagswasserkanal (NWK) - Graz, Ziegelstraße}

Das Einzugsgebiet dieser Probenahmestelle liegt im Norden von Graz und weist eine kanalisierte Fläche von 41,9 ha auf, wovon 12,9 ha befestigt und davon 8,3 ha befestigt und an den Niederschlagswasserkanal (NWK) angeschlossen sind. Die Bebauungsstruktur kann als eher ländlich geprägte Wohngegend in Stadtrandlage beschrieben werden (großer Anteil von Einfamilienhäusern, keine größeren Gewerbe- oder Industriebetriebe, die größte Verkehrsbelastung ist durch einen Kreuzungs- bereich gegeben, welchen ca. 23.000 Kfz pro Tag passieren). Die Probenahme erfolgte hier unmittelbar vor der Einleitung der Niederschlagswasserkanalisation in den Stufenbach direkt im Niederschlagswasserkanal (DN 500). Im Kanal war während des gesamten Beprobungszeitraums ein relativ konstanter Fremdwasserabfluss bei Trockenwetterbedingungen von $0,5-3 \mathrm{~L} / \mathrm{s}$ bei $1-3 \mathrm{~cm}$ Wasserstand festzustellen.

\subsection{Mischwasserkanal (MWK) - Graz, Mischwasserüberlauf R05 (MÜ- R05)}

Das Einzugsgebiet dieser Probenahmestelle liegt im Westen von Graz und weist eine kanalisierte Fläche von 615 ha auf, wovon 232 ha befestigt und davon 147 ha befestigt und an den Kanal angeschlossen sind (in etwa 17mal größer als das Einzugsgebiet des Niederschlagswasserkanals). Als Sonderbauwerke sind im Einzugsgebiet ein Stauraumkanal ohne Entlastung mit einem Volumen von $2300 \mathrm{~m}^{3}$ sowie 
ein Mischwasserüberlaufbauwerk (MÜR05) am Ende des Einzugsgebiets vor der Einleitung in den rechten Hauptsammelkanal in Richtung Kläranlage Graz-Gössendorf vorhanden. Die Bebauungsstruktur kann als gemischtes Gewerbe- und Wohngebiet mit 19.500 EinwohnerInnen beschrieben werden. Im Einzugsgebiet befinden sich ein Pharmakonzern und eine Brauerei (beides Indirekteinleiter), stark befahrene Einzugsstraßen, eine Straßenbahn- und zwei Eisenbahnlinien.

Die Probenahme erfolgte hier direkt in der Mischwasserkammer des MÜR05, da an dieser Stelle der Gesamtabfluss aus dem Einzugsgebiet erfasst werden konnte. Während des einjährigen Beprobungszeitraums traten im Mischwasserkanal (MWK) bei Trockenwetterbedingungen Wasserstandshöhen von $16-28 \mathrm{~cm}$ bei einem Abfluss von $10-70 \mathrm{~L} / \mathrm{s}$ auf. Bei einer Wasserstandshöhe von ca. $90 \mathrm{~cm}$ in der Mischwasserkammer beginnt der Mischwasserüberlauf über die Überlaufschwelle in die Mur zu entlasten.

\section{Beschreibung der verwendeten Probenahmegeräte und deren Parametrierung}

\subsection{Niederschlagswasserkanal (NWK) -} Graz, Ziegelstraße

Die Entnahme von Abwasserproben im Ereignisfall erfolgte an dieser Probenahmestelle mithilfe von zwei automatischen, ungekühlten Probenahmegeräten der Fa. Endress + Hauser $(\mathrm{E}+\mathrm{H})$ vom Typ Liquiport 2010 CSP44, die beide zeitproportional und in Serie betrieben wurden. Die Ansaugung der Teilproben wurde dabei mit einer Peristaltikpumpe (Schlauchquetschpumpe) durchgeführt. Die Ansaughöhe aus dem Niederschlagswasserkanal betrug nur ca. $1,5 \mathrm{~m}$. Abhängig von einem vordefinierten Wasserstand im Kanal wurde die Probenahme automatisch aktiviert (Start der Probenahme anfänglich bei $10 \mathrm{~cm}$ und dann schließlich bei $9 \mathrm{~cm}$ Wasserstandshöhe, Ende bei anfänglich $6 \mathrm{~cm}$ und schließlich bei $7 \mathrm{~cm}$ Wasserstandshöhe). Zur Erfassung des Durchflusses und der Wasserstandshöhe im Kanal wurde eine Ultraschallsonde der Fa. Nivus GmbH vom Typ „POA Korrelations-Keilsensor" an der Kanalsohle installiert. Die entnommenen Teilproben wurden in Behältern mit $24 \mathrm{PE}$ Flaschen mit je 1,0L Fassungsvermö- gen ohne Kühlung bis zur Abholung zwischengespeichert.

Damit das verwendete automatische Probenahmegerät bei längeren Niederschlagsereignissen nicht überfüllt wurde, wurden an dieser Probenahmestelle zwei idente Probenahmegeräte in Serie geschaltet, wovon das erste mit einem Probenahmeintervall von $4 \mathrm{~min}$ und das zweite Gerät dann nach der vollständigen Befüllung des ersten mit einem Probenahmeintervall von $8 \mathrm{~min}$ betrieben wurde, wodurch an dieser Probenahmestelle maximale EreignisProbenahmedauern von $24 \mathrm{~h}$ erreicht werden konnten. Als Teilprobenvolumen wurden $180 \mathrm{~mL}$ gewählt, wonach jede der 1-L-PE-Probenahmeflaschen jeweils 5 Teilproben aufnehmen konnte. Die Befüllung einer Probenahmeflasche dauerte bei einem Probenahmeintervall von $4 \mathrm{~min} 20 \mathrm{~min}$ bzw. bei $8 \mathrm{~min} 40 \mathrm{~min}$.

\subsection{Mischwasserkanal (MWK) - Graz, Mischwasserüberlauf R05 (MÜ- R05)}

Die Entnahme der Mischwasserproben erfolgte an dieser Probenahmestelle aufgrund der leicht abbaubaren Inhaltsstoffe in der Mischwassermatrix mithilfe eines automatischen, aktiv gekühlten Probenahmegeräts der Fa. Maxx GmbH vom Typ TP5C. Die Ansaugung wurde dabei mit einer Membranpumpe (Vakuumpumpe) durchgeführt. Die Ansaughöhe aus der Mischwasserkammer des MÜ-R05 betrug ca. 6,5 m. Das Probenahmegerät wurde zeitproportional mit einem Standardintervall von 4 min betrieben. Abhängig von einem vordefinierten Wasserstand in der Mischwasserkammer über der Trockenwetterrinne wurde die Probenahme automatisch aktiviert (Start der Probenahme bei $50 \mathrm{~cm}$ Wasserstandshöhe, Ende bei $45 \mathrm{~cm}$ Wasserstandshöhe). Zur Erfassung des Durchflusses und der Wasserstandshöhe im Zulaufkanal zur Kammer des MÜ-R05 wurde ein Radarmessgerät der Fa. Marsh McBirney vom Typ FloDar verwendet, das am Kanalscheitel etwa $25 \mathrm{~m}$ vor dem Probenahmeort im zulaufenden Mischwasserkanal installiert wurde. Zusätzlich dazu wurde die Wasserstandshöhe in der Mischwasserkammer auch noch durch einen zweiten Sensor der Fa. Vega vom Typ Vegason 61 in unmittelbarer Nähe des Ansaugpunkts der Probenahme gemessen. Dieser Sensor arbeitet nach dem Prinzip der Ultraschall-Laufzeitmessung und diente der Ansteuerung des automatischen Probenahmegeräts. Die entnommenen Proben wurden in einem aktiv gekühlten Probenahmeuntersatz mit 24 PE-Flaschen mit jeweils 1,0 L Fassungsvermögen gesammelt.

Damit das verwendete automatische Probenahmegerät bei längeren Niederschlagsereignissen nicht überfüllt wurde, wurde an dieser Probenahmestelle mit einer sog. Programmverkettung gearbeitet, bei der ab der Befüllung der 13. von 24 Probenahmeflaschen automatisch auf ein doppelt so langes Probenahmeintervall von 8 min umgeschaltet wurde, wodurch die maximale Ereignis-Probenahmedauer an dieser Probenahmestelle auf insgesamt $12 \mathrm{~h}$ ausgedehnt werden konnte. Als Teilprobenvolumen wurden auch hier $180 \mathrm{~mL}$ gewählt, wonach jede der 1-L-PE-Probenahmeflaschen jeweils 5 Teilproben aufnehmen konnte. Die Befüllung dauerte bei einem Probenahmeintervall von $4 \mathrm{~min} 20 \mathrm{~min}$ bzw. bei $8 \mathrm{~min} 40 \mathrm{~min}$.

\section{Angewandte Methodik bei den automatischen Probenahmen}

Um möglichst alle Niederschlagsereignisse eines Jahres vollständig und mengenproportional beproben zu können, erfolgte in Graz die automatische Teilprobenentnahme an beiden Probenahmestellen zunächst zeitproportional, d.h., dass der Zeitabstand (Teilprobenintervall) zwischen den einzelnen Teilprobenentnahmen konstant war und dafür möglichst geringe konstante Zeitintervalle von i.d.R. 4 min gewählt wurden. Erst in einem zweiten Schritt wurden die Teilproben im Nachgang an die Probenahmen und in Abhängigkeit von der jeweiligen Abflussganglinie der Ereignisse mengenproportional zu den jeweiligen Ereignisproben händisch zusammengemischt. Dabei wurde die Variante gewählt, dass die Mischung der Teilproben aus den 24 bzw. 48 Einzelflaschen der verwendeten Probenahmegeräte abhängig vom zugehörigen Abflussvolumen jeder Einzelflasche erfolgte, was nach Ort et al. (2010) die genaueste Art einer mengenproportionalen Probenahme darstellt.

Nach der manuellen Herstellung der mengenproportionalen Ereignisproben wurden diese anschließend anteilig von den beiden abgeschätzten Jahresabflussvolumina zu jeweils zwei Halbjahresmischproben kumuliert und möglichst bald nach den Ereignissen kumulativ eingefroren. 
Tab. 1 Abflussbedingungen bei Trockenwetter und Aktivierung der Probenahmen bei Niederschlagswasserabfluss an der Probenahmestelle NWK-Graz, Ziegelstraße

\begin{tabular}{lllll|}
\hline Probenahmestelle & $\begin{array}{l}\text { Wasserstände bei } \\
\text { TW-Beding. }\end{array}$ & $\begin{array}{l}\text { Abfluss bei } \\
\text { TW-Beding. }\end{array}$ & \multicolumn{2}{l|}{$\begin{array}{l}\text { Probenahme-Aktivierung } \\
\text { Ein }\end{array}$} \\
$\begin{array}{l}\text { Graz-Ziegelstr. } \\
\text { (NWK) }\end{array}$ & $1-3 \mathrm{~cm}$ & $0,5-3 \mathrm{~L} / \mathrm{s}$ & $10 / 9 \mathrm{~cm}^{\mathrm{a}}$ & $6 / 7 \mathrm{~cm}^{\mathrm{b}}$ \\
\hline $\begin{array}{l}\text { aAb 13.04.2018 } \\
\text { bAb 12.03.2018 }\end{array}$ & & & & \\
\hline
\end{tabular}

Tab. 2 Diegemessenen und beprobten Abflussvolumina an der NWK-Probenahmestelle Graz-Ziegelstraße inkl. der gemessenen Jahresniederschlagshöhe im Zeitraum 01.10.2017-30.09.2018

\begin{tabular}{|c|c|c|c|c|c|c|}
\hline \multirow[t]{2}{*}{ Probenahmestelle } & \multicolumn{5}{|c|}{ Abflussvolumina } & \multirow[t]{2}{*}{ NS-Höhe im UG } \\
\hline & Insgesamt & TW & NW & NW beprobt & Insg. beprobt (inkl. FW) & \\
\hline \multirow{2}{*}{$\begin{array}{l}\text { Graz-Ziegelstr. } \\
\text { (NWK) }\end{array}$} & $103.260 \mathrm{~m}^{3} / \mathrm{a}$ & $57.424 \mathrm{~m}^{3} / \mathrm{a}$ & $45.836 \mathrm{~m}^{3} / \mathrm{a}$ & $26.939 \mathrm{~m}^{3} / \mathrm{a}$ & $28.289 \mathrm{~m}^{3} / \mathrm{a}$ & \multirow[t]{2}{*}{$760 \mathrm{~mm}$} \\
\hline & $100 \%$ & $56 \%$ & $44 \%$ & $26 \%$ & $27 \%$ & \\
\hline
\end{tabular}

\begin{tabular}{|l|l|}
\hline Fehlereinfluss bei den Ereignisproben & Anzahl \\
\hline kein & 50 \\
\hline gering & 7 \\
\hline mittel & 8 \\
\hline groß & 11 \\
\hline
\end{tabular}

Abb. 2 Bewertung des Fehlereinflusses bei den 76 Ereignisproben an der NWKProbnahmestelle Graz-Ziegelstraße

In Abb. 1 ist beispielhaft eine automatisierte Ereignis-Auswertung für die MWK-Probenahmestelle „Graz - MÜR05“ und das Ereignis mit der ID 64 dargestellt. Im linken Teil der Auswertung sind die Messdaten der Kanalabflussraten $(\mathrm{L} / \mathrm{s})$, der Wasserstandshöhen (cm) und der Niederschlagsintensitäten $(\mathrm{mm} / \mathrm{min})$ in Kombination mit den Zeitpunkten und der Parametrierung der Teilprobenahmen dargestellt.

Der rechte Teil der Auswertung enthält eine statistische Auswertung zum theoretischen Gesamt-Probenvolumen, den Mischungsvolumina für alle betroffenen Probenahmeflaschen zur Erstellung der mengenproportionalen Ereignismischprobe und dem einzufrierenden Probenvolumen zur Erstellung der mengenproportionalen Jahresmischprobe (abhängig vom abgeschätzten Jahres-Abflussvolumen, der angestrebten Jahres-Probenmenge und dem relativen Ereignisanteil am Jahresabflussvolumen). Im rechten unteren Teil der Auswertungen finden sich noch Statistiken zur Ereignisdauer und ergänzende Kennwerte zur Probenahme.

Sofern ausreichend Probenvolumen von den Ereignisproben übrig blieb, wurden diese Ereignisproben in Graz zusätzlich auch noch auf die Standardparameter CSB, $\mathrm{CSB}_{\mathrm{mf}}$, AFS, AFS63 und Leitfähigkeit untersucht. Bei einigen
Ereignisproben wurde zusätzlich auch noch der Glühverlust bestimmt.

\section{Ergebnisse der beiden Jahresbeprobungen}

Aufgrund des Trockenwetterabflusses im Mischwasserkanal und des relativ konstanten Fremdwasserabflusses im Niederschlagswasserkanal bei Trockenwetter mussten für das Aktivieren der Probenahmegeräte jeweils Ein- und Ausschaltpunkte vorgegeben werden, welche jeweils über Wasserstandssensoren getriggert wurden. Diese wurden nach einer Voranalysenphase über die vorherrschende hydraulische Dynamik an den beiden Probenahmestellen definiert und stellten letztendlich einen Kompromiss zwischen dem Ziel einer möglichst vollständigen Erfassung des Niederschlagswasser- bzw. Mischwasserabflusses über ein gesamtes Jahr und den praktischen Erfordernissen an eine möglichst gesicherte Jahresbetreuung rund um die Uhr dar.

\subsection{Niederschlagswasserkanal (NWK) - Graz, Ziegelstraße}

Eine eingehende Trockenwetter-Fremdwasseranalyse an der Probenahmestelle NWK Graz - Ziegelstraße ergab Wasserstandshöhen von $1-3 \mathrm{~cm}$ und dazugehörige Trockenwetterabflüsse von 0,5-3 L/s, die in der Tab. 1 während des einjährigen Beprobungszeitraums (01.10.2017-30.09.2018) an der NWKProbenahmestelle Graz - Ziegelstraße inkl. der Ein- und Ausschaltpunkte für die Aktivierung der Probenahmen zusammengefasst sind.

Auf Basis dieser Vorgaben für die Aktivierung der automatischen Probenahmen sind in der nachfolgenden Tab. 2 die insgesamt während des einjährigen Beprobungszeitraums (01.10.2017-30.09.2018) an der NWKProbenahmestelle - Graz, Ziegelstraße gemessenen Abflussvolumina, die Trockenwetter- und Niederschlagswasseranteile, die insgesamt beprobten $\mathrm{Ab}$ flussvolumina sowie die Jahresniederschlagshöhe in dem Untersuchungsgebiet dargestellt.

Dabei wurden hier insgesamt 76 Niederschlagswasserereignisse beprobt, woraus die beiden nachfolgenden $\mathrm{ku}-$ mulierten, mengenproportionalen Halbjahresmischproben generiert wurden:

- 13,36 L Halbjahresprobe (01.10.201705.05.2018) Graz - Ziegelstraße,

- 20,73 L Halbjahresprobe (06.05.201830.09.2018) Graz - Ziegelstraße.

Dabei war die Messdatenverfügbarkeit bei den Abflussraten im NWK und bei den Niederschlagsintensitäten einer Niederschlagsmessstation im Einzugsgebiet mit $100 \%$ vollständig gegeben.

Eine im Nachgang an die Probenahmen durchgeführte qualitative Ereignisvalidierung ergab die in der Abb. 2 zusammengefassten Ergebnisse.

Bewertungskriterien dabei waren Fehler bei der Ereignisabgrenzung, bei der Datenverfügbarkeit, bei der automatischen Probenahme und bei der mengenproportionalen Mischung der Proben.

In der Abb. 3 sind die 76 Ereignisse inklusive ihrer o.a. Bewertungen nach ihrem beprobten Abflussvolumen geordnet und der kumulative Anteil der Ereignisvolumina am beprobten Jahresniederschlagswasservolumen in \% dargestellt.

Beispielhaft seien hierfür die schlechteren Bewertungen für die beiden größten aufgetretenen Ereignisse (ID 45 vom 13.06.2018 und ID 22 vom 16.04.2018) näher beschrieben: Beim Ereignis mit 


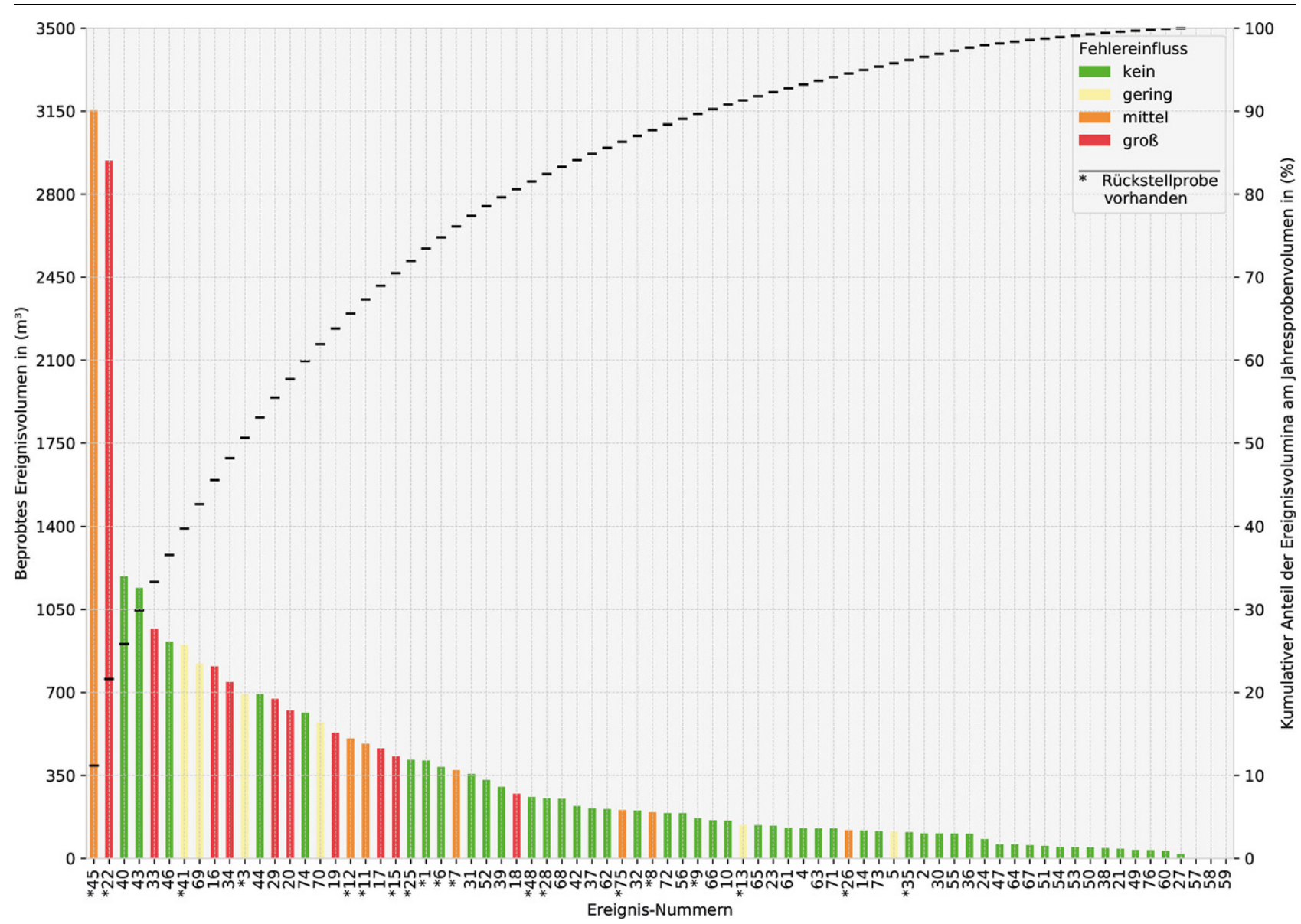

Abb. 3 Darstellung der 76 bewerteten Einzelereignisse an der NWK-Probenahmestelle Graz-Ziegelstraße nach der Größe des beprobten Abflussvolumens sortiert inkl. der kumulativen Anteile am Jahresprobenvolumen

der ID 45 führte eine um ca. $25 \mathrm{~min}$ zu späte Zuweisung des ProbenahmeStart- und Probenahme-Endzeitpunkts zu einer Übergewichtung bei der Mischung der ersten Probenahme-Flaschen von $18 \%$ anstatt von $12 \%$, wodurch ein vermeintlich mit den ersten Probenahmeflaschen erfasster Spülstoß etwas überproportional gewichtet wurde. Außerdem wurde dadurch das beprobte Ereignisabflussvolumen mit $3100 \mathrm{~m}^{3}$ anstatt mit $3700 \mathrm{~m}^{3}$ ermittelt. Beim Ereignis mit der ID $22 \mathrm{kam}$ es bei zwei Probenahmeflaschen zu Ausfällen bei der Teilprobenentnahme, wodurch die nachfolgenden Probenahmeflaschen falsch gemischt wurden. Eine genaue Quantifizierung dieser aufgetretenen Fehler während der beiden Ereignisse auf die kumulierte Halbjahresmischprobe ist nicht möglich.

\subsection{Mischwasserkanal - Graz, Mischwasserüberlauf R05 (MÜ- R05)}

Eine eingehende Trockenwetteranalyse an der Probenahmestelle MWK Graz, MÜ-R05 ergab Wasserstandshöhen von $16-28 \mathrm{~cm}$ und dazugehörige Trockenwetterabflüsse von $10-70 \mathrm{~L} / \mathrm{s}$, die in Tab. 3 für den einjährigen Beprobungszeitraum (01.10.2017-30.09.2018) an der Probenahmestelle MWK - Graz, MÜ-R05 inkl. der Ein- und Ausschaltpunkte für die Aktivierung der Probenahmen zusammengefasst sind.

Auf Basis dieser Vorgaben für die Aktivierung der automatischen Probenahmen sind in der nachfolgenden Tab. 4 die insgesamt während des einjährigen Beprobungszeitraums (01.10.2017-30.09.2018) an der MWKProbenahmestelle - Graz, MÜ-R05 gemessenen Abflussvolumina, die Trockenwetter- und Niederschlagswasseranteile, die insgesamt beprobten Abflussvolumina sowie die Jahresnie- derschlagshöhe in dem Untersuchungsgebiet dargestellt.

Insgesamt wurden 73 Mischwasserereignisse beprobt, woraus die beiden nachfolgenden aufkumulierten, mengenproportionalen Halbjahresmischproben generiert wurden:

- 25,05 L Halbjahresprobe (01.10.201705.05.2018) Graz - MÜ-R05,

- 39,61 L Halbjahresprobe (06.05.201830.09.2018) Graz - MÜ-R05.

Die Messdatenverfügbarkeit bei den Abflussraten im MWK war bis auf die beiden von der Abflussspitze her größten Ereignisse mit den Ereignis-Nummern 16 vom 16.04.2018 (mit lokal bis zu $162 \mathrm{~mm}$ Niederschlag innerhalb von $3 \mathrm{~h}$ am Rande des Einzugsgebiets) und 44 vom 13.06.2018 vollständig gegeben. Nachdem bei diesen beiden Ereignissen die Mischwasserkammer des MÜ-R05 unter Druck ging, versagten dabei temporär leider sowohl die Ultraschall-Wasserstandshöhenmessung in der Mischwasserkammer als auch 
Tab. 3 Abflussbedingungen bei Trockenwetter und Aktivierung der Probenahmen bei Mischwasserabfluss an der Probenahmestelle MWK-Graz, MÜ-R05

\begin{tabular}{llllll} 
Probenahmestelle & $\begin{array}{l}\text { Wasserstände bei } \\
\text { TW-Beding. }\end{array}$ & $\begin{array}{l}\text { Abfluss bei } \\
\text { TW-Beding. }\end{array}$ & \multicolumn{2}{l}{$\begin{array}{l}\text { Probenahme-Aktivierung } \\
\text { Ein }\end{array}$} & Aus \\
\hline $\begin{array}{l}\text { Graz-R05 } \\
\text { (MWK) }\end{array}$ & $16-28 \mathrm{~cm}$ & $10-70 \mathrm{~L} / \mathrm{s}$ & $50 \mathrm{~cm}$ & $45 \mathrm{~cm}$ \\
\hline
\end{tabular}

Tab. 4 Diegemessenen und beprobten Abflussvolumina an der MWK-Probenahmestelle-Graz, MÜ-R05 inkl. der gemessenen Jahresniederschlagshöhe im Zeitraum 01.10.2017-30.09.2018

\begin{tabular}{llllll|l} 
Probenahmestelle & $\begin{array}{l}\text { Abflussvolumina } \\
\text { Insgesamt }\end{array}$ & TW & NW & NW beprobt & Insg. beprobt (inkl. MW) \\
\hline $\begin{array}{l}\text { Graz-R05 } \\
\text { (MWK) }\end{array}$ & $1.854 .905 \mathrm{~m}^{3} / \mathrm{a}$ & $1.266 .563 \mathrm{~m}^{3} / \mathrm{a}$ & $588.342 \mathrm{~m}^{3} / \mathrm{a}$ & $377.998 \mathrm{~m}^{3} / \mathrm{a}$ & $405.540 \mathrm{~m}^{3} / \mathrm{a}$ \\
\hline
\end{tabular}

\begin{tabular}{|l|l|}
\hline Fehlereinfluss bei den Ereignisproben & Anzahl \\
\hline kein & 56 \\
\hline gering & 8 \\
\hline mittel & 4 \\
\hline groß & 5 \\
\hline
\end{tabular}

Abb. 4 Bewertung des Fehlereinflusses bei den 73 Ereignisproben an der MWKProbenahmestelle Graz-MÜ-R05

das Radar-Durchflussmesssystem im Zulaufkanal zum MÜ-R05. Dadurch stoppte während dieser Zeit zum einen die automatische Teilprobenentnahme und zum anderen konnte dadurch auch das Abflussvolumen dieser beiden Ereignisse messtechnisch nicht vollständig erfasst werden. Ersatzweise wurden dann im Nachgang die Abflussganglinien dieser beiden Ereignisse mit einem vorhandenen kalibrierten hydrodynamischen Abflussmodell und dem real gemessenen Niederschlagsverlauf an der Niederschlagsmessstation GrazStraßgang-ZAMG berechnet und daraus dann auch das Abflussvolumen für diese beiden Ereignisse abgeschätzt.

Im Gegensatz dazu war die Messdatenverfügbarkeit bei den Niederschlagsintensitäten der Niederschlagsmessstation Graz-Straßgang-ZAMG im Einzugsgebiet über den gesamten Beprobungszeitraum hinweg mit $100 \%$ vollständig gegeben.

Die im Nachgang an die Probenahmen durchgeführte Ereignisvalidierung der 73 Ereignisproben an der MWKProbenahmestelle Graz - R05 ergab hinsichtlich der aufgetretenen Fehler bei der Erstellung der Ereignismischproben die in der Abb. 4 zusammengefassten Ergebnisse.

Bewertungskriterien dabei waren wieder - analog zur Probenahmestelle NWK Graz - Ziegelstraße - Fehler bei der Ereignisabgrenzung, bei der Datenverfügbarkeit, bei der automatischen
Probenahme und bei der mengenproportionalen Mischung der Proben.

In der Abb. 5 sind die 73 Ereignisse inklusive ihrer o. a. Bewertung nach ihrem beprobten Abflussvolumen nach geordnet und der kumulative Anteil der Ereignisvolumina am beprobten Jahresabflussvolumen in \% dargestellt.

Beispielhaft sei hierfür die schlechtere Bewertung für das größte aufgetretene Ereignis mit der ID 16 vom 16.04.2018 mit insgesamt 94,3 mm Niederschlag in $3 \mathrm{~h}$ näher beschrieben. Nachdem bei diesem Ereignis sowohl die Mischwasserkammer als auch der zulaufende Kanal unter Druck gingen, fielen sowohl die Wasserstandshöhenmessung in der Mischwasserkammer, welche das Probenahmegerät triggerte, als auch das Radar-Durchflussmengenmesssystem im Zulaufkanal aus. Ersteres führte dazu, dass temporär und solange die Kammer unter Druck war, keine Teilprobenentnahmen erfolgten und Zweiteres dazu, dass die Abflussspitze während dieser Periode messtechnisch nicht erfasst werden konnte. Für eine Abschätzung des Abflussvolumens während dieses Ereignisses bediente man sich eines vorhandenen hydrodynamischen Modells vom Einzugsgebiet des MÜ-R05, mit dem das Ereignis mit dem gemessenen Niederschlagsverlauf nachsimuliert werden konnte. Das führte bei diesem über $17 \mathrm{~h}$ langen Ereignis dazu, dass von den insgesamt mit Simulation abgeschätzten $89.000 \mathrm{~m}^{3}$ Abflussvolumen nur die ersten $12 \mathrm{~h}$ mit $82.000 \mathrm{~m}^{3}$ beprobt werden konnten, dabei aber während der Ereignisspitze für einen Zeitraum von 01:20 h keine Teilprobenentnahmen stattfanden, in welcher ca. $35.000 \mathrm{~m}^{3}$ Mischwasser unbeprobt abflossen. Eine genaue Quantifizierung all dieser aufgetretenen Fehlereinflüsse während dieses Ereignisses auf die kumulierte Halbjahresmischprobe ist nicht mög- lich, führte aber letztendlich $\mathrm{zu}$ der sehr schlechten Bewertung dieses Ereignisses mit einem hohen, bewerteten Fehlereinfluss.

\subsection{Ergebnisse der}

Standardparameterbestimmungen an den beiden Probenahmestellen

Sofern bei der Erstellung der einzelnen mengenproportionalen Ereignisproben und nach Abzug der Ereignisaliquote genügend Probenvolumen für die Bestimmung von Abwasserstandardparametern übrig blieb, wurden in Graz von diesen mengenproportionalen Ereignisproben die Standardparameter $\mathrm{CSB}, \mathrm{CSB}_{\mathrm{mf}}$, AFS, AFS63 und Leitfähigkeit untersucht. Die Ergebnisse dieser Untersuchungen sind in der nachfolgenden Abb. 6 zusammengefasst.

Die Mediane der Glühverluste und der AFS63-Anteile am AFS-Gesamt betrugen bei den untersuchten MWK-Proben $46 \%$ bzw. $54 \%$ und bei den untersuchten NWK-Proben $22 \%$ bzw. $70 \%$.

\section{Erfahrungen bei der Probenahme und Diskussion der Probenahme-Ergebnisse}

Die gewählte Methodik zur Generierung mengenproportionaler Ereignisproben war sehr arbeitsaufwendig, da diese nach den zeitproportionalen automatischen Probenahmen jeweils manuell anhand der Abflussganglinien zusammengemischt werden mussten, führte jedoch über das gesamte Beprobungsjahr gesehen zu einer sehr hohen Ausbeute an erfolgreich generierten Ereignisproben. Sie hatte auch den großen Vorteil, dass die automatischen Teilprobenentnahmen unabhängig von den Abflussmessungen sind, wodurch die Teilprobenentnahmen auch bei Ausfall der Abflussmengenmessung durchgeführt werden können. 


\section{Originalarbeit}

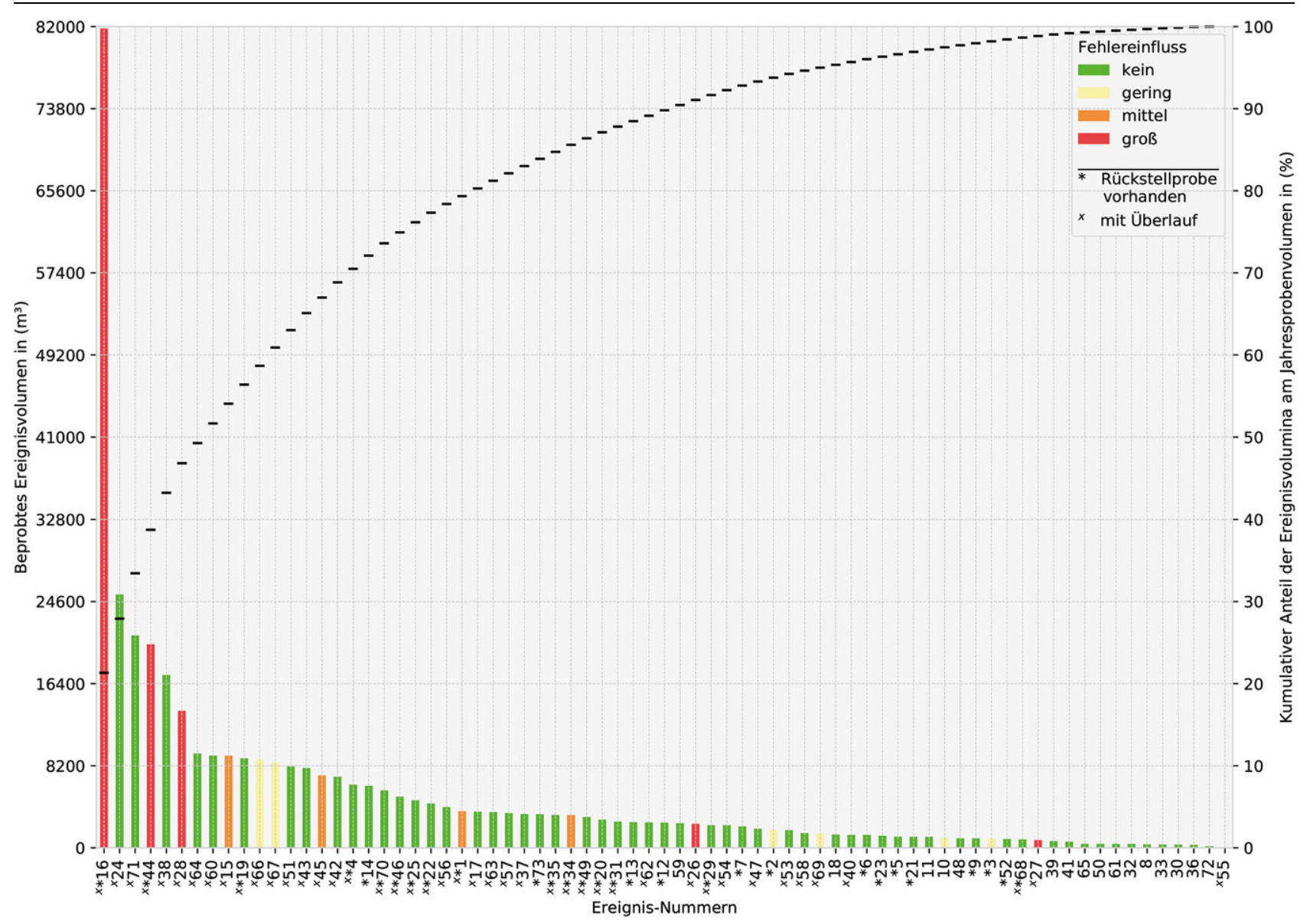

Abb. 5 Darstellung der 73 bewerteten Ereignisse an der MWK-Probenahmestelle Graz-MÜ-R05 nach der Größe des beprobten Abflussvolumens gereiht inkl. der kumulativen Anteile am Jahresprobenvolumen
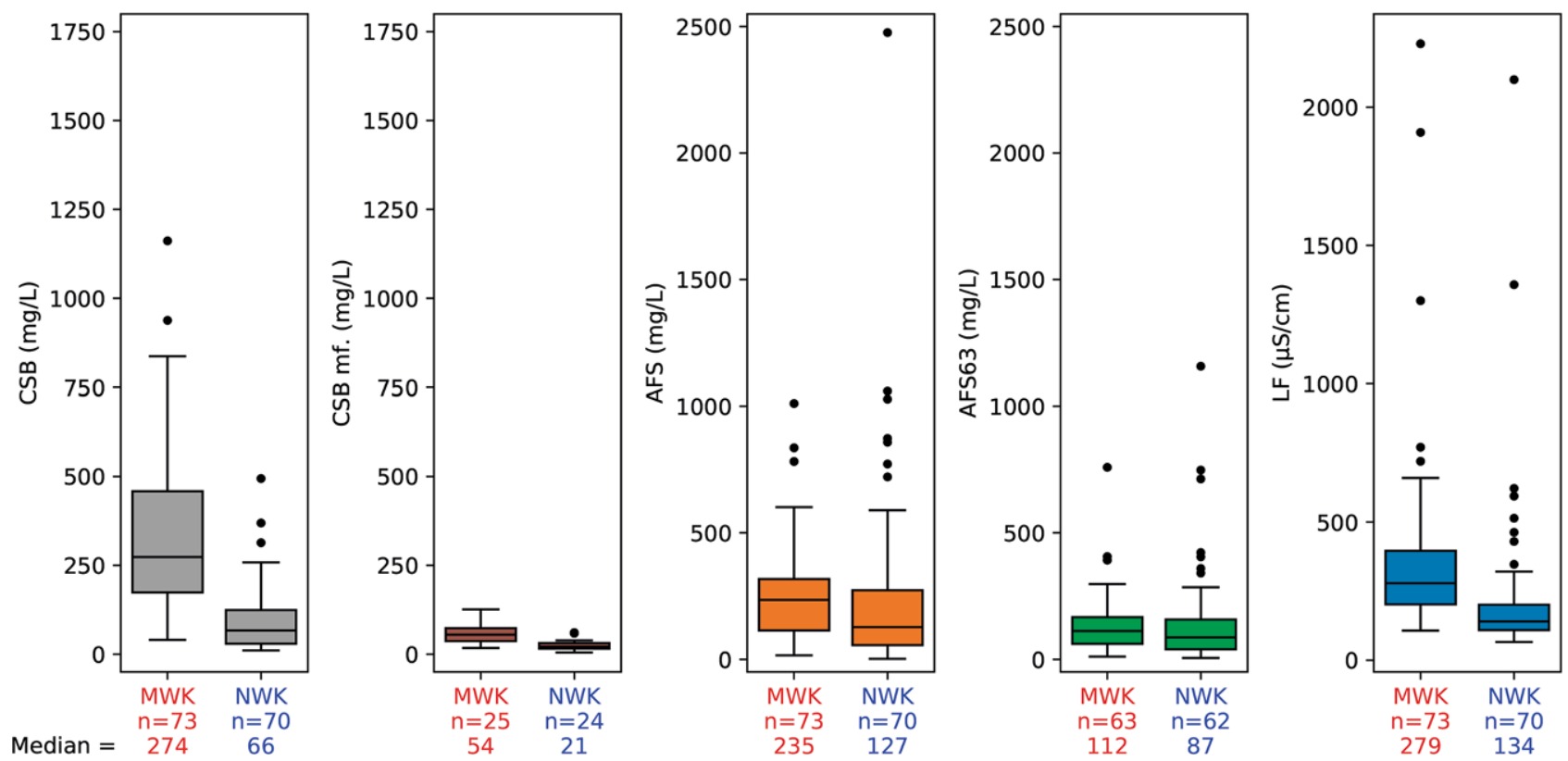

Abb. 6 Ergebnisse der Standardparameter-Untersuchungen in den Mischwasserproben (MWK) und in den Niederschlagswasserproben (NWK) inkl. der Anzahl der durchgeführten Analysen und ermittelten Mediane 
Weiters konnten zwei fehlende Abflussganglinien bei den zwei aufgetretenen Extremereignissen an der Probenahmestelle MWK - MÜ-R05 mittels hydrodynamischer Simulation rekonstruiert werden und damit dann auch die Mischungstabellen für das Herstellen der mengenproportionalen Ereignisproben im Nachhinein noch erzeugt werden. Nachdem bei diesen beiden Extremereignissen aber auch die Mischwasserkammer unter Druck ging, versagten während dieser beiden Ereignisse nicht nur das installierte Radar-Durchflussmesssystem, sondern auch die in der Mischwasserkammer installierte Ultraschall-Wasserstandssonde zum Triggern des Probenahmegeräts, was dazu führte, dass während der Einstauperiode in der Kammer auch keine Teilproben entnommen wurden. Dies könnte durch Verwendung einer piezometrischen Wasserstandsmessung verhindert werden, welche die Druckhöhen in der Mischwasserkammer auch noch bei Einstau der Kammer zuverlässig messen würde. Das ist insofern von großer Bedeutung, da diese beiden Extremereignisse auch einen überproportional großen Anteil am Niederschlagswasserjahresabfluss aufwiesen.

Nachteilig an der angewandten Methodik ist sicherlich die erforderliche Probenteilung der inhomogenen Proben aus jeder der befüllten Probenahmeflaschen anhand der zu erstellenden Mischungstabellen, was insbesondere bei partikelhaltigen Rohabwasserproben immer fehlerbehaftet ist.

Die Benachrichtigung der diensthabenden TU-Graz-Projektmitarbeiter erfolgte im Ereignisfall über eine automatisierte E-Mail-Alarmierung, aus welcher sowohl der Start- als auch der Endzeitpunkt der Probenahmen ersichtlich war. Auf Basis dieser Benachrichtigungen wurde der zeitliche Ablauf der Probenabholungen von den Probenahmestellen organisiert. Dabei bringt die gewählte zeitproportionale Teilprobenentnahme auch den Vorteil mit sich, dass man nach dem Alarmierungsbeginn der automatischen Teilprobenentnahmen genau weiß, wann das Probenahmegerät vollkommen befüllt sein wird und daher spätestens entleert werden muss, um es für das nachfolgende Ereignis wieder betriebsbereit zu machen.

Durch die angewandte Programmverkettung beim MWK-Probenahmegerät bzw. durch das In-Serie-Konfigurieren von zwei identen Probenahmegeräten beim NWK konnte ein vorzeitiges Ende der automatischen Teilprobenentnahmen weitestgehend verhindert werden und damit fast alle aufgetretenen Ereignisse innerhalb des Beprobungsjahrs vollständig und mengenproportional beprobt werden.

Wie zu erwarten war, schwankten die Ergebnisse der durchgeführten Standardparameter-Untersuchungen bei den einzelnen Ereignissen über große Bereiche und können auch nicht direkt auf andere Einzugsgebiete übertragen werden. Aufgrund der großen Anzahl an untersuchten Einzelereignissen liefern die resultierenden Mediane aber sehr repräsentative Hinweise über die mittleren $\mathrm{zu}$ erwartenden Konzentrationen aus den beiden untersuchten Einzugsgebieten in Graz. Generell lagen diese bei den untersuchten Standardparametern im Mischwasserkanal höher als im Niederschlagswasserkanal.

Danksagung Die dargestellten Ergebnisse entstammen dem Forschungsprojekt „(T)EMPEST“ (Erfassung von Emissionen ausgewählter Spurenstoffe aus Kanalsystemen und Handlungsoptionen zu deren Minderung und Optimierung einer alternativen Nachweismethode für Kunststoffpartikel in Wasserproben), GZ B601390, welches dankenswerterweise vom Bundesministerium für Nachhaltigkeit und Tou- rismus in Österreich gefördert wird. Unser Dank gilt aber auch der Holding Graz Wasserwirtschaft, die uns bei der Ausstattung und beim Betrieb der beiden Probenahmestellen in Graz tatkräftigst unterstützt hat, sowie unserem Labor-, Messtechnik- und ITTeam am Institut für Siedlungswasserwirtschaft und Landschaftswasserbau der TU Graz, ohne dessen Einsatz das einjährige Probenahmeprogramm an den zwei Probenahmestellen in Graz nicht möglich gewesen wäre.

Funding Open access funding provided by Graz University of Technology.

Open Access Dieser Artikel wird unter der Creative Commons Namensnennung 4.0 International Lizenz veröffentlicht, welche die Nutzung, Vervielfältigung, Bearbeitung, Verbreitung und Wiedergabe in jeglichem Medium und Format erlaubt, sofern Sie den/die ursprünglichen Autor(en) und die Quelle ordnungsgemäß nennen, einen Link zur Creative Commons Lizenz beifügen und angeben, ob Änderungen vorgenommen wurden.

Die in diesem Artikel enthaltenen Bilder und sonstiges Drittmaterial unterliegen ebenfalls der genannten Creative Commons Lizenz, sofern sich aus der Abbildungslegende nichts anderes ergibt. Sofern das betreffende Material nicht unter der genannten Creative Commons Lizenz steht und die betreffende Handlung nicht nach gesetzlichen Vorschriften erlaubt ist, ist für die oben aufgeführten Weiterverwendungen des Materials die Einwilligung des jeweiligen Rechteinhabers einzuholen.

Weitere Details zur Lizenz entnehmen Sie bitte der Lizenzinformation auf http://creativecommons.org/licenses/ by/4.0/deed.de. 


\section{Originalarbeit}

Clara, M., Ertl, T., Giselbrecht, G., Gruber, G., Hofer, T., Humer, F., Kretschmer, F., Koll, L. Scheffknecht, C., Weiß, S. und Windhofer, G. (2014): SCHTURM - Spurenstoffemissionen aus Siedlungsgebieten und von Verkehrsflächen. Studie im Auftrag des Bundesministeriums für Land- und Forstwirtschaft, Umwel und Wasserwirtschaft. Wien, Österreich. https:// www.bmnt.gv.at/service/publikationen/wasser/ Spurenstoffemissionen-aus-Siedlungsgebietenund-von-Verkehrsflaechen.html (Letzter Zugriff am 12.01.2020).

Huber, M., Welker, A. und Helmreich, B. (2015): Belastung von Verkehrsflächenabflüssen mit Schwermetallen - ein europäischer Vergleich. gwf-Wasser Abwasser 9/2015, S. 896-909.
Launay, M. (2017): Organic micropollutants in urban wastewater systems during dry and wet weather - Occurrence, spatio-temporal distribution and emissions to surface waters. Dissertation. Universität Stuttgart.

MKULNV (2016): Entwicklung und Stand der Abwasserbeseitigung in Nordrhein-Westfalen. 17. Auflage. Düsseldorf: Ministerium für Klimaschutz, Umwelt, Landwirtschaft, Natur- und Verbraucherschutz des Landes Nordrhein-Westfalen.

Ort, C., Lawrence, M. G., Reungoat, J. and Mueller, J. F. (2010): Sampling for PPCPs in Wastewater Systems: Comparison of Different Sampling Modes and Optimization Strategies. Environ. Sci. Technol. 2010, 44, p. 6289-6296.
Wicke, D., Matzinger, A. und Rouault, P. (2016): OgRe - Relevanz organischer Spurenstoffe im Regenwasserabfluss Berlins. Kompetenzzentrum Wasser Berlin. Berlin, Deutschland. https://www.kompetenz-wasser.de/wp-content uploads/2017/11/abschlussbericht_ogre_final_ rev2.pdf (Letzter Zugriff am 18.08.2019).

Hinweis des Verlags Der Verlag bleibt in Hinblick auf geografische Zuordnungen und Gebietsbezeichnungen in veröffentlichten Karten und Institutsadressen neutral. 